\title{
TACRED Revisited: A Thorough Evaluation of the TACRED Relation Extraction Task
}

\author{
Christoph Alt Aleksandra Gabryszak Leonhard Hennig \\ German Research Center for Artificial Intelligence (DFKI) \\ Speech and Language Technology Lab \\ \{christoph.alt, aleksandra.gabryszak, leonhard.hennig\}@dfki.de
}

\begin{abstract}
TACRED (Zhang et al., 2017) is one of the largest, most widely used crowdsourced datasets in Relation Extraction (RE). But, even with recent advances in unsupervised pretraining and knowledge enhanced neural RE, models still show a high error rate. In this paper, we investigate the questions: Have we reached a performance ceiling or is there still room for improvement? And how do crowd annotations, dataset, and models contribute to this error rate? To answer these questions, we first validate the most challenging $5 \mathrm{~K}$ examples in the development and test sets using trained annotators. We find that label errors account for $8 \%$ absolute F1 test error, and that more than $50 \%$ of the examples need to be relabeled. On the relabeled test set the average F1 score of a large baseline model set improves from 62.1 to 70.1. After validation, we analyze misclassifications on the challenging instances, categorize them into linguistically motivated error groups, and verify the resulting error hypotheses on three state-of-the-art RE models. We show that two groups of ambiguous relations are responsible for most of the remaining errors and that models may adopt shallow heuristics on the dataset when entities are not masked.
\end{abstract}

\section{Introduction}

Relation Extraction (RE) is the task of extracting relationships between concepts and entities from text, where relations correspond to semantic categories such as per:spouse, org:founded_by or org:subsidiaries (Figure 1). This makes RE a key part of many information extraction systems, and its performance determines the quality of extracted facts for knowledge base population ( $\mathrm{Ji}$ and Grishman, 2011), or the quality of answers in question answering systems (Xu et al., 2016). Standard benchmarks such as SemEval 2010 Task 8 (Hendrickx et al., 2010) and the more recent TACRED

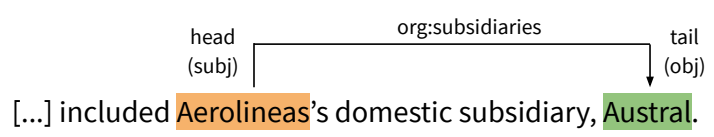

Figure 1: Relation example from TACRED. The sentence contains the relation org:subsidiaries between the head and tail organization entities 'Aerolineas' and 'Austral'.

(Zhang et al., 2017) are essential to evaluate new RE methods and their limitations, and to establish open challenges.

TACRED is one of the largest and most widely used RE datasets. It contains more than 106k examples annotated by crowd workers. The methods best performing on the dataset use some form of pre-training to improve RE performance: finetuning pre-trained language representations (Alt et al., 2019; Shi and Lin, 2019; Joshi et al., 2019) or integrating external knowledge during pre-training, e.g. via joint language modelling and linking on entity-linked text (Zhang et al., 2019; Peters et al., 2019; Baldini Soares et al., 2019); with the last two methods achieving a state-of-the-art performance of $71.5 \mathrm{~F} 1$. While this performance is impressive, the error rate of almost $30 \%$ is still high. The question we ask in this work is: Is there still room for improvement, and can we identify the underlying factors that contribute to this error rate? We analyse this question from two separate viewpoints: (1) to what extent does the quality of crowd based annotations contribute to the error rate, and (2) what can be attributed to dataset and models? Answers to these questions can provide insights for improving crowdsourced annotation in RE, and suggest directions for future research.

To answer the first question, we propose the following approach: We first rank examples in the development and test sets according to the misclas- 
sifications of $49 \mathrm{RE}$ models and select the top 5k instances for evaluation by our linguists. This procedure limits the manual effort to only the most challenging examples. We find that a large fraction of the examples are mislabeled by the crowd. Our first contribution is therefore a extensively relabeled TACRED development and test set.

To answer the second question, we carry out two analyses: (1) we conduct a manual explorative analysis of model misclassifications on the most challenging test instances and categorize them into several linguistically motivated error categories; (2) we formulate these categories into testable hypotheses, which we can automatically validate on the full test set by adversarial rewriting - removing the suspected cause of error and observing the change in model prediction (Wu et al., 2019). We find that two groups of ambiguous relations are responsible for most of the remaining errors. The dataset also contains clues that are exploited by models without entity masking, e.g. to correctly classify relations even with limited access to the sentential context.

We limit our analysis to TACRED, but want to point out that our approach is applicable to other RE datasets as well. We make the code of our analyses publicly available. ${ }^{1}$ In summary, our main contributions in this paper are:

- We validate the $5 \mathrm{k}$ most challenging examples in the TACRED development and test sets, and provide a revised dataset ${ }^{2}$ that will improve the accuracy and reliability of future RE method evaluations.

- We evaluate the most challenging, incorrectly predicted examples of the revised test set, and develop a set of 9 categories for common RE errors, that will also aid evaluation on other datasets.

- We verify our error hypotheses on three stateof-the-art RE models and show that two groups of ambiguous relations are responsible for most of the remaining errors and that models exploit cues in the dataset when entities are unmasked.

\footnotetext{
${ }^{1}$ https://github.com/DFKI-NLP/tacrev

${ }^{2}$ Due to licensing restrictions, we can not publish the dataset but instead provide a patch to the original TACRED.
}

\begin{tabular}{lcc}
\hline Split & \# Examples & \# Neg. examples \\
\hline Train & 68,124 & 55,112 \\
Dev & 22,631 & 17,195 \\
Test & 15,509 & 12,184 \\
\hline
\end{tabular}

Table 1: TACRED statistics per split. About $79.5 \%$ of the examples are labeled as no_relation.

\section{The TACRED Dataset}

The TAC Relation Extraction $D$ ataset $^{3}$, introduced by Zhang et al. (2017), is a fully supervised dataset of sentence-level binary relation mentions. It consists of $106 \mathrm{k}$ sentences with entity mention pairs collected from the TAC KBP ${ }^{4}$ evaluations 20092014, with the years 2009 to 2012 used for training, 2013 for development, and 2014 for testing. Each sentence is labeled with one of 41 person- and organization-oriented relation types, e.g. per:title, org:founded, or the label no_relation for negative instances. Table 1 summarizes key statistics of the dataset.

All relation labels were obtained by crowdsourcing, using Amazon Mechanical Turk. Crowd workers were shown the example text, with head (subject) and tail (object) mentions highlighted, and asked to select among a set of relation label suggestions, or to assign no_relation. Label suggestions were limited to relations compatible with the head and tail types. ${ }^{5}$

The data quality is estimated as relatively high by Zhang et al. (2017), based on a manual verification of 300 randomly sampled examples (93.3\% validated as correct). The inter-annotator kappa label agreement of crowd workers was moderate at $\kappa=0.54$ for 761 randomly selected mention pairs.

\section{An Analysis of TACRED Label Errors}

In order to identify the impact of potentially noisy, crowd-generated labels on the observed model performance, we start with an analysis of TACRED's label quality. We hypothesize that while comparatively untrained crowd workers may on average produce relatively good labels for easy relation mentions, e.g. those with obvious syntactic and/or

\footnotetext{
${ }^{3}$ https: / / catalog.ldc.upenn.edu/ LDC2018T24

${ }^{4}$ https://tac.nist.gov/2017/KBP/index. html

${ }^{5}$ See the supplemental material provided by Zhang et al. (2017) for details of the dataset creation and annotation process.
} 
lexical triggers, or unambiguous entity type signatures such as per:title, they may frequently err on challenging examples, e.g. highly ambiguous ones or relation types whose scope is not clearly defined.

An analysis of the complete dataset using trained annotators would be prohibitively expensive. We therefore utilize a principled approach to selecting examples for manual analysis (Section 3.1). Based on the TAC-KBP annotation guidelines, we then validate these examples (Section 3.2), creating new Dev and Test splits where incorrect annotations made by crowd workers are revised (Section 3.3).

\subsection{Data Selection}

Since we are interested in identifying potentially incorrectly labeled examples, we implement a selection strategy which is based upon ordering examples by the difficulty of predicting them correctly. ${ }^{6}$ We use a set of 49 different RE models to obtain predictions on the development and test sets, and rank each example according to the number of models predicting a different relation label than the ground truth. ${ }^{7}$ Intuitively, examples with large disagreement, between all models or between models and the ground truth, are either difficult, or incorrectly annotated.

We select the following examples for validation: (a) Challenging - all examples that were misclassified by at least half of the models, and (b) Control - a control group of (up to) 20 random examples per relation type, including no_relation, from the set of examples classified correctly by at least 39 models. The two groups cover both presumably hard and easy examples, and allow us to contrast validation results based on example difficulty. In total we selected 2,350 (15.2\%) Test examples and 3,655 (16.2\%) Dev examples for validation. Of these, 1,740 (Test) and 2,534 (Dev) were assigned a positive label by crowd workers.

\subsection{Human Validation}

We validate the selected examples on the basis of the TAC KBP guidelines. ${ }^{8}$ We follow the approach of Zhang et al. (2017), and present each example by showing the example's text with highlighted head and tail spans, and a set of relation label suggestions. We differ from their setup by showing

\footnotetext{
${ }^{6}$ A similar approach was used e.g. by Barnes et al. (2019).

${ }^{7}$ See the supplemental material for details on the models, training procedure, hyperparameters, and task performance.

${ }^{8}$ https: / / tac.nist.gov/2014/KBP/ ColdStart/guidelines/TAC_KBP_2014_Slot_ Descriptions_V1.4.pdf
}

more label suggestions to make the label choice less restrictive: (a) the original, crowd-generated ground truth label, (b) the set of labels predicted by the models, (c) any other relation labels matching the head and tail entity types, and (d) no_relation. The suggested positive labels are presented in an alphabetical order and are followed by no_relation, with no indication of a label's origin. Annotators are asked to assign no_relation or up to two positive labels from this set. A second label was allowed only if the sentence expressed two relations, according to the guidelines, e.g. per:city_of_birth and per:city_of_residence. Any disagreements are subsequently resolved by a third annotator, who is also allowed to consider the original ground truth label. All annotators are educated in general linguistics, have extensive prior experience in annotating data for information extraction tasks, and are trained in applying the task guidelines in a trial annotation of 500 sentences selected from the development set.

\subsection{The Revised TACRED Dev and Test Sets}

Table 2 shows the results of the validation process. In total, the annotators revised 960 (49.9\%) of the Challenging Test examples, and 1,610 (52.1\%) of the Challenging Dev examples, a very large fraction of label changes for both dataset splits. Revision rates for originally positive examples are lower at $47.3 \%$ (Test) and $49.1 \%$ (Dev). Approximately $57 \%$ of the negative examples were relabeled with a positive relation label (not shown). Two labels were assigned to only $3.1 \%$ of the Test, and $2.4 \%$ of the Dev examples. The multi-labeling mostly occurs with location relations, e.g. the phrase "[Gross] head:per, a 60-year-old native of [Potomac] $]_{\text {tail:city }}$ " is labeled with per:city_of_birth and per:city_of_residence, which is justified by the meaning of the word native.

As expected, the revision rate in the Control groups is much lower, at $8.9 \%$ for Test and $8.1 \%$ for Dev. We can also see that the fraction of negative examples is approximately one-third in the Challenging group, much lower than the dataset average of $79.5 \%$. This suggests that models have more difficulty predicting positive examples correctly.

The validation inter-annotator agreement is shown in Table 3 . It is very high at $\kappa_{\text {Test }}=0.87$ and $\kappa_{D e v}=0.80$, indicating a high annotation quality. For both Test and Dev, it is higher for the easier Control groups than for the Challenging 


\begin{tabular}{|c|c|c|c|c|}
\hline & \multicolumn{2}{|c|}{ Dev } & \multicolumn{2}{|l|}{ Test } \\
\hline & Challenging & Control & Challenging & Control \\
\hline \# Examples (\# positive) & $3,088(1,987)$ & $567(547)$ & $1,923(1,333)$ & 427 (407) \\
\hline \# Revised (\# positive) & $1,610 \quad(976)$ & $46 \quad(46)$ & $960 \quad(630)$ & $38 \quad(38)$ \\
\hline \# Revised (\% positive) & $52.1(49.1)$ & $8.1(8.4)$ & $49.9(47.3)$ & $8.9(9.3)$ \\
\hline
\end{tabular}

Table 2: Re-annotation statistics for TACRED Dev and Test splits.

\begin{tabular}{lcc|cc}
\hline & \multicolumn{2}{c}{ Dev } & \multicolumn{2}{c}{ Test } \\
IAA & $\mathrm{H} 1, \mathrm{H} 2$ & $\mathrm{H}, \mathrm{C}$ & $\mathrm{H} 1, \mathrm{H} 2$ & $\mathrm{H}, \mathrm{C}$ \\
\hline Challenging & 0.78 & 0.43 & 0.85 & 0.44 \\
Control & 0.87 & 0.95 & 0.94 & 0.96 \\
\hline All & 0.80 & 0.53 & 0.87 & 0.55 \\
\hline
\end{tabular}

Table 3: Inter-Annotator Kappa-agreement for the relation validation task on TACRED Dev and Test splits ( $\mathrm{H} 1, \mathrm{H} 2$ = human re-annotators, $\mathrm{H}=$ revised labels, $\mathrm{C}=$ original TACRED crowd-generated labels).

groups. In contrast, the average agreement between our annotators and the crowdsourced labels is much lower at $\kappa_{\text {Test }}=0.55, \kappa_{\text {Dev }}=0.53$, and lowest for Challenging examples (e.g., $\kappa_{\text {Test }}=0.44$ ).

Frequently erroneous crowd labels are per:cities_of_residence, org:alternate_names, and per:otherfamily. Typical errors include mislabeling an example as positive which does not express the relation, e.g. labeling "[Alan Gross] head:per was arrested at the [Havana] $]_{t a i l: l o c}$ airport." as per:cities_of_residence, or not assigning a positive relation label, e.g. per:other_family

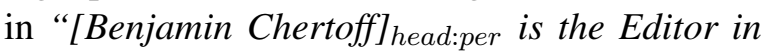
Chief of Popular Mechanics magazine, as well as the cousin of the Director of Homeland Security, [Michael Chertoff] tail:per". Approximately 49\% of the time an example's label was changed to no_relation during validation, $36 \%$ of the time from no_relation to a positive label, and the remaining $15 \%$ it was changed to or extended with a different relation type.

To measure the impact of dataset quality on the performance of models, we evaluated all 49 models on the revised test split. The average model F1 score rises to $70.1 \%$, a major improvement of $8 \%$ over the $62.1 \%$ average $\mathrm{F} 1$ on the original test split, corresponding to a $21.1 \%$ error reduction.

Discussion The large number of label corrections and the improved average model performance show that the quality of crowdsourced annotations is a major factor contributing to the overall error rate of models on TACRED. Even though our selection strategy was biased towards examples challenging for models, the large proportion of changed labels suggests that these examples were difficult to label for crowd workers as well. To put this number into perspective - Riedel et al. (2010) showed that, for a distantly supervised dataset, about $31 \%$ of the sentence-level labels were wrong, which is less than what we observe here for human-supervised data. $^{9}$

The low quality of crowd-generated labels in the Challenging group may be due to their complexity, or due to other reasons, such as lack of detailed annotation guidelines, lack of training, etc. It suggests that, at least for Dev and Test splits, crowdsourcing, even with crowd worker quality checks as used by Zhang et al. (2017), may not be sufficient to produce high quality evaluation data. While models may be able to adequately utilize noisily labeled data for training, measuring model performance and comparing progress in the field may require an investment in carefully labeled evaluation datasets. This may mean, for example, that we need to employ well-trained annotators for labeling evaluation splits, or that we need to design better task definitions and task presentations setups as well as develop new quality control methods when using crowd-sourced annotations for complex NLP tasks like RE.

\section{An Analysis of Model Errors}

After revising the dataset, we focus on the two open questions: which of the remaining errors can be attributed to the models, and what are potential reasons for misclassifications? To answer these, we first create an annotation task instructing the

\footnotetext{
${ }^{9}$ Riedel et al.'s estimate is an average over three relations with 100 randomly sampled examples each, for similar news text. Two of the relations they evaluated, nationality and place_of_birth, are also contained in TACRED, the third is contains (location).
} 
linguists to annotate model misclassifications with their potential causes (Section 4.1). We then categorize and analyze the causes and formulate testable hypotheses that can be automatically verified ( $\mathrm{Sec}$ tion 4.2). For the automatic analysis, we implemented a baseline and three state-of-the-art models (Section 4.3).

\subsection{Misclassification Annotation}

The goal of the annotation is to identify possible linguistic aspects that cause incorrect model predictions. We first conduct a manual exploratory analysis on the revised Control and Challenging test instances that are misclassified by the majority of the 49 models. Starting from single observations, we iteratively develop a system of categories based on the existence, or absence, of contextual and entity-specific features that might mislead the models (e.g. entity type errors or distracting phrases). Following the exploration, we define a final set of categories, develop guidelines for each, and instruct two annotators to assign an error category to each misclassified instance in the revised test subset. In cases where multiple categories are applicable the annotator selected the most relevant one. As in the validation step, any disagreements between the two annotators are resolved by a third expert.

\subsection{Error Hypotheses Formulation and Adversarial Rewriting}

In a next step, we extend the misclassification categories to testable hypotheses, or groups, that are verifiable on the whole dataset split. For example, if we suspect a model to be distracted by an entity in context of same type as one of the relation arguments, we formulate a group has_distractor. The group contains all instances, both correct and incorrect, that satisfy a certain condition, e.g. there exists at least one entity in the sentential context of same type as one of the arguments. The grouping ensures that we do not mistakenly prioritize groups that are actually well-handled on average. We follow the approach proposed by Wu et al. (2019), and extend their Errudite framework ${ }^{10}$ to the relation extraction task. After formulating a hypothesis, we assess the error prevalence over the entire dataset split to validate whether the hypothesis holds, i.e. the group of instances shows an above average error rate. In a last step, we test the error hypothesis explicitly by adversarial rewriting of a group's ex-

\footnotetext{
${ }^{10}$ https://github.com/uwdata/errudite
}

amples, e.g. by replacing the distracting entities and observing the models' predictions on the rewritten examples. In our example, if the has_distractor hypothesis is correct, removing the entities in context should change the prediction of previously incorrect examples.

\subsection{Models}

We evaluate our error hypotheses on a baseline and three of the most recent state-of-the-art RE models. None of the models were part of the set of models used for selecting challenging instances (Section 3.1), so as not to bias the automatic evaluation. As the baseline we use a single layer CNN (Zeng et al., 2014; Nguyen and Grishman, 2015) with max-pooling and 300-dimensional GloVe (Pennington et al., 2014) embeddings as input. The state-of-the-art models use pre-trained language models (LM) fine-tuned to the RE task and include: TRE (Alt et al., 2019), which uses the unidirectional OpenAI Generative Pre-Trained Transformer (GPT) (Radford et al., 2018); SpanBERT (Joshi et al., 2019), which employs a bidirectional LM similar to BERT (Devlin et al., 2019) but is pre-trained on span-level; and KnowBERT (Peters et al., 2019), which is an extension to BERT that integrates external knowledge. In particular, we use KnowBERT-W+W, which is trained by joint entity linking and language modelling on Wikipedia and WordNet.

\section{Model Error and Dataset Analysis}

In this section, we present our analysis results, providing an answer to the question: which of the remaining errors can be attributed to the models, and what are the potential reasons for these errors? We first discuss the findings of our manual misclassification analysis (Section 5.1), followed by the results of the automatic analysis (Section 5.2).

\subsection{Model Error Categories}

Table 4 summarizes the linguistic misclassification categories we developed. We distinguish between errors resulting from (1) relation argument errors, and (2) context misinterpretation. ${ }^{11}$ The category relation argument errors refers to misclassifications resulting from incorrectly assigned

\footnotetext{
${ }^{11}$ The manual analysis focused on the sentence semantics, and left aspects such such as sentence length, distance between entities, etc. for the automatic analysis, which can handle the analysis of surface features more effectively.
} 


\begin{tabular}{|c|c|c|c|}
\hline Error Type & Examples & Prediction & Freq. \\
\hline \multicolumn{4}{|l|}{ Arguments } \\
\hline Span & This is a tragic day for the Australian [Defence Force $]_{\text {head:org }}\left([\mathrm{ADF}]_{\text {tail:org }}\right)$ & org:alt._nam & 12 \\
\hline Entity Type & $[\text { Christopher Bollyn }]_{\text {head:per }}$ is an [independent $]_{\text {tail:religion }}$ journalist & per:religion & 31 \\
\hline & $\begin{array}{l}\text { The company, which [Baldino }]_{\text {head:org }} \text { founded in }[1987]_{\text {tail:date }} \text { sells a variety } \\
\text { of drugs }\end{array}$ & org:founded & \\
\hline \multicolumn{4}{|l|}{ Context } \\
\hline Inverted Args & $\begin{array}{l}{[\text { Ruben van Assouw }]_{h e a d: p e r}, \text { who had been on safari with his } 40 \text {-year-old father }} \\
{[\text { Patrick }]_{\text {tail:per }}, \text { mother Trudy }, 41 \text {, and brother Enzo }, 11 \text {. }}\end{array}$ & per:children & 25 \\
\hline Wrong Args & $\begin{array}{l}\text { Authorities said they ordered the detention of Bruno's wife }, \text { [Dayana } \\
\text { Rodrigues }]_{\text {tail:per }} \text {, who was found with [Samudio] } \frac{\text { head:per's baby }}{\text {. }}\end{array}$ & per:spouse & 109 \\
\hline Ling. Distractor & $\begin{array}{l}\text { In May, [he }]_{\text {head:per }} \text { secured } \$ 96,972 \text { in working capital from [GE Healthcare } \\
\text { Financial Services] }]_{\text {tail:org }} \text {. }\end{array}$ & per:employ._of & 35 \\
\hline Factuality & $\begin{array}{l}\left.\text { [Ramon] }]_{\text {head:per }} \text { said he hoped to one day become an [astronaut }\right]_{\text {head:title }} \\
\text { Neither he nor [Aquash] }\end{array}$ & $\begin{array}{l}\text { per:title } \\
\text { per:origin }\end{array}$ & 11 \\
\hline Relation Def. & $\begin{array}{l}\text { [Zhang Yinjun }]_{\text {tail:per }} \text {, spokesperson with one of China 's largest charity organi- } \\
\text { zation, the }[\text { China Charity Federation }]_{\text {head:org }}\end{array}$ & org:top_mem. & 96 \\
\hline Context Ignored & [Bibi] $]_{\text {head:per }}$, a mother of [five $]_{\text {tail:number }}$, was sentenced this month to death . & per:age & 52 \\
\hline \multirow[t]{2}{*}{ No Relation } & {$[\mathrm{He}]_{\text {head:per }}$ turned a gun on himself committing [suicide] tail:causeofdeath } & no_relation & 646 \\
\hline & & Total & 1017 \\
\hline
\end{tabular}

Table 4: Misclassification types along with sentence examples, relevant false predictions, and error frequency. The problematic sentence parts are underlined (examples may be abbreviated due to space constraints).

entity spans or entity types of arguments. We always labeled type annotation errors, but tolerated minor span annotation errors if they did not change the interpretation of the relation or the entity.

The category context misinterpretation refers to cases where the sentential context of the arguments is misinterpreted by the model. We identify the following context problems: (1) Inverted arguments: the prediction is inverse to the correct relation, i.e. the model's prediction would be correct if head and tail were swapped. (2) Wrong arguments: the model incorrectly predicts a relation that holds between head or tail and an un-annotated entity mention in the context, therefore misinterpreting one annotated argument. (3) Linguistic distractor: the example contains words or phrases related to the predicted relation, however they do not connect to any of the arguments in a way justifying the prediction. (4) Factuality: the model ignores negation, speculation, future tense markers, etc. (5) Context ignored: the example does not contain sufficient linguistic evidence for the predicted relation except for the matching entity types. (6) Relation definition: the predicted relation could be inferred from the context using common sense or world knowledge, however the inference is prohibited by the guidelines (e.g. the spokesperson of an organization is not a top member/employee, or a work location is not a pointer to the employee's residence). (7) No Relation: the model incorrectly predicts no_relation even though there is sufficient linguistic evidence for the relation in the sentential context.

Discussion The relation label predicted most frequently across the 49 models disagreed with the ground truth label of the re-annotated Challenging and Control Test groups in 1017 (43.3\%) of the cases. The inter-annotator agreement of error categories assigned to these examples is high at $\kappa_{\text {Test }}=0.83\left(\kappa_{\text {Test }}=0.67\right.$ if the category No Relation is excluded).

Argument errors accounted for only 43 (4.2\%) misclassifications, since the entities seem to be mostly correctly assigned in the dataset. In all entity type misclassification cases except one, the errors originate from false annotations in the dataset itself.

Context misinterpretation caused 974 (95.8\%) false predictions. No relation is incorrectly assigned in $646(63.6 \%)$ of misclassified instances, even though the correct relation is often explicitly and unambiguously stated. In 134 (13.2\%) of the erroneous instances the misclassification resulted from inverted or wrong argument assignment, i.e. the predicted relation is stated, however the arguments are inverted or the predicted relation involves an entity other than the annotated one. In $96(9.4 \%)$ instances the error results from TAC KBP guidelines prohibiting specific inferences, affecting most often the classification of the relations per:cities_of_residence and 

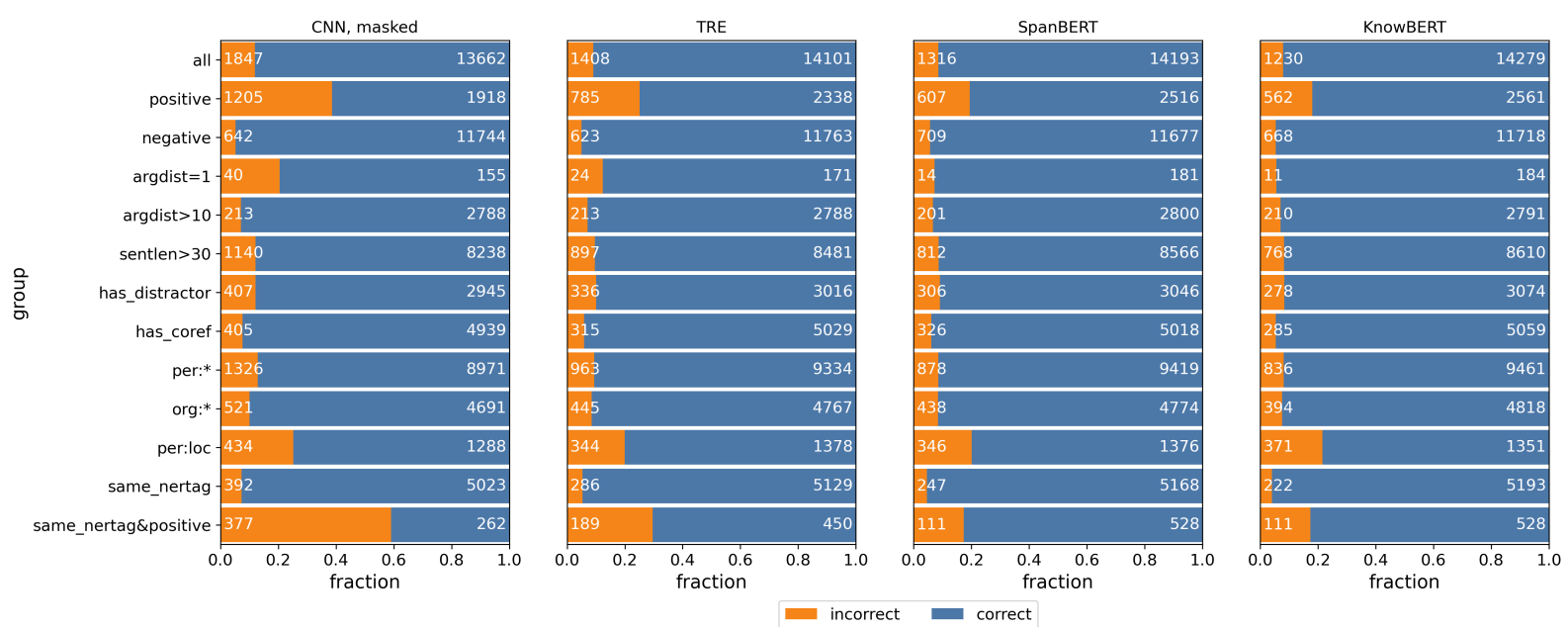

Figure 2: Error rates for different groups (example subsets) on the revised TACRED test set, for four different models. The bars show the number and fraction of correctly (blue) and incorrectly (orange) predicted examples in the given group. KnowBert, as the best-performing model, has the lowest error rates across most groups. Error rates for per:loc, same nertag \&positive are higher for all models than the model error rate on the complete test set (all), highlighting examples for further error analysis and potential model improvements.

org:top_member/employee. Furthermore, in 52 $(5.1 \%)$ of the false predictions models seem to ignore the sentential context of the arguments, i.e. the predictions are inferred mainly from the entity types. Sentences containing linguistic distractors accounted for 35 (3.4\%) incorrect predictions. Factuality recognition causes only 11 errors $(1.1 \%)$ However, we assume that this latter low error rate is due to TACRED data containing an insufficient number of sentences suitable for extensively testing a model's ability to consider the missing factuality of relations.

\subsection{Automatic Model Error Analysis}

For the automatic analysis, we defined the following categories and error groups:

- Surface structure - Groups for argument distance (argdist $=1$, argdist $>10$ ) and sentence length (sentlen $>30$ )

- Arguments - Head and tail mention NER type (same nertag, per:*, org:*,per:loc), and pronominal head/tail (has_coref)

- Context - Existence of distracting entities (has_distractor)

- Ground Truth - Groups conditioned on the ground truth (positive, negative, same_nertag\&positive)

Figure 2 shows the error rates of different groups on the revised TACRED test set. The plot shows error rates across four representative models. Each chart displays groups on the y-axis, and the fraction and number of correct (blue) vs. incorrect (orange) instances in the respective group on the $\mathrm{x}$-axis. The average error rate of each model on the full test set is shown for reference in the top-most column titled all. Groups with higher than average error rate may indicate characteristics of examples that make classification difficult. On the other hand, groups with lower than average error rate comprise examples that the given model performs especially well on.

What is the error rate for different groups? In Figure 2, we can see that KnowBERT has the lowest error rate on the full test set $(7.9 \%)$, and the masked CNN model the highest (11.9\%). SpanBERT's and TRE's error rates are in between the two. Overall, all models exhibit a similar pattern of error rates across the groups, with KnowBERT performing best across the board, and the CNN model worst. We can see that model error rates e.g. for the groups has_distractor, argdist $>10$, and has_coref do not diverge much from the corresponding overall model error rate. The presence of distracting entities in the context therefore does not seem to be detrimental to model performance. Similarly, examples with a large distance between the relation arguments, or examples where co-referential information is required, are generally predicted correctly. On the other hand, we can see that all models have above-average error rates for the group positive, 
its subgroup same_nertag \& positive, and the group per:loc. The above-average error rate for positive may be explained by the fact that the dataset contains much fewer positive than negative training instances, and is hence biased towards predicting no_relation. A detailed analysis shows that the groups per:loc and same nertag\&positive are the most ambiguous. per:loc contains relations such as per:cities_of_residence, per:countries_of_residence and per:origin, that may be expressed in a similar context but differ only in the fine-grained type of the tail argument (e.g. per:city vs. per:country). In contrast, same_nertag contains all person-person relations such as per:parents, per:children and per:other_family, as well as e.g. org:parent and org:subsidiaries that involve the same argument types (per:per vs. org:org) and may be only distinguishable from context.

How important is context? KnowBERT and SpanBERT show about the same error rate on the groups per:loc and same_nertag\&positive. They differ, however, in which examples they predict correctly: For per:loc, $78.6 \%$ are predicted by both models, and $21.4 \%$ are predicted by only one of the models. For same_nertag\&positive, $12.8 \%$ of the examples are predicted by only of the models. The two models thus seem to identify complementary information. One difference between the models is that KnowBERT has access to entity information, while SpanBERT masks entity spans.

To test how much the two models balance context and argument information, we apply rewriting to alter the instances belonging to a group and observe the impact on performance. We use two strategies: (1) we remove all tokens outside the span between head and tail argument (outside), and (2) we remove all tokens between the two arguments (between). We find that SpanBERT's performance on per:loc drops from $62.1 \mathrm{~F} 1$ to 57.7 (outside) and 43.3 (between), whereas KnowBERT's score decreases from $63.7 \mathrm{~F} 1$ to 60.9 and 50.1, respectively. On same_nertag\&positive, we observe a drop from $89.2 \mathrm{~F} 1$ to 58.2 (outside) and 47.7 (between) for SpanBERT. KnowBERT achieves a score of 89.4 , which drops to 83.8 and 49.0. The larger drop in performance on same_nertag\&positive suggests that SpanBERT, which uses entity masking, focuses more on the context, whereas KnowBERT focuses on the entity content because the model has access to the arguments. Surprisingly, both models show similar
Original Revised Weighted

Model

\begin{tabular}{llll}
\hline CNN, masked & 59.5 & 66.5 & 34.8 \\
TRE & 67.4 & 75.3 & 48.8 \\
SpanBERT & 70.8 & 78.0 & 61.9 \\
KnowBERT & 71.5 & 79.3 & 58.7 \\
\hline
\end{tabular}

Table 5: Test set F1 score on TACRED, our revised version, and weighted by difficulty (on revised). The weight per instance is determined by the number of incorrect predictions in our set of $49 \mathrm{RE}$ models. The result suggests that SpanBERT better generalizes to more challenging examples, e.g. complex sentential context.

performance on the full test set (Table 5). This suggests that combining both approaches may further improve RE performance.

Should instance difficulty be considered? Another question is whether the dataset contains instances that can be solved more easily than others, e.g. those with simple patterns or patterns frequently observed during training. We assume that these examples are also more likely to be correctly classified by our baseline set of 49 RE models.

To test this hypothesis, we change the evaluation setup and assign a weight to each instance based on the number of correct predictions. An example that is correctly classified by all 49 baseline models would receive a weight of zero - and thus effectively be ignored - whereas an instance misclassified by all models receives a weight of one. In Table 5, we can see that SpanBERT has the highest score on the weighted test set (61.9 F1), a $16 \%$ decrease compared to the unweighted revised test set. KnowBERT has the second highest score of $58.7,3 \%$ less than SpanBERT. The performance of TRE and CNN is much worse at 48.8 and $34.8 \mathrm{~F} 1$, respectively. The result suggests that SpanBERT's span-level pre-training and entity masking are beneficial for RE and allow the model to generalize better to challenging examples. Given this observation, we propose to consider an instance's difficulty during evaluation.

\section{Related Work}

Relation Extraction on TACRED Recent RE approaches include PA-LSTM (Zhang et al., 2017) and GCN (Zhang et al., 2018), with the former combining recurrence and attention, and the latter leveraging graph convolutional neural networks. 
Many current approaches use unsupervised or semi-supervised pre-training: fine-tuning of language representations pre-trained on token-level (Alt et al., 2019; Shi and Lin, 2019) or span-level (Joshi et al., 2019), fine-tuning of knowledge enhanced word representations that are pre-trained on entity-linked text (Zhang et al., 2019; Peters et al., 2019), and "matching the blanks" pre-training (Baldini Soares et al., 2019).

Dataset Evaluation Chen et al. (2016) and Barnes et al. (2019) also use model results to assess dataset difficulty for reading comprehension and sentiment analysis. Other work also explores bias in datasets and the adoption of shallow heuristics on biased datasets in natural language inference (Niven and Kao, 2019) and argument reasoning comprehension (McCoy et al., 2019).

Analyzing trained Models Explanation methods include occlusion or gradient-based methods, measuring the relevance of input features to the output (Zintgraf et al., 2017; Harbecke et al., 2018), and probing tasks (Conneau et al., 2018; Kim et al., 2019) that probe the presence of specific features e.g. in intermediate layers. More similar to our approach is rewriting of instances (Jia and Liang, 2017; Ribeiro et al., 2018) but instead of evaluating model robustness we use rewriting to test explicit error hypotheses, similar to Wu et al. (2019).

\section{Conclusion and Future Work}

In this paper, we conducted a thorough evaluation of the TACRED RE task. We validated the 5k most challenging examples in development and test set and showed that labeling is a major error source, accounting for $8 \%$ absolute F1 error on the test set. This clearly highlights the need for careful evaluation of development and test splits when creating datasets via crowdsourcing. To improve the evaluation accuracy and reliability of future RE methods, we provide a revised, extensively relabeled TACRED. In addition, we categorized model misclassifications into 9 common RE error categories and observed that models are often unable to predict a relation, even if it is expressed explicitly. Models also frequently do not recognize argument roles correctly, or ignore the sentential context. In an automated evaluation we verified our error hypotheses on the whole test split and showed that two groups of ambiguous relations are responsible for most of the remaining errors. We also showed that models adopt heuristics when en- tities are unmasked and proposed that evaluation metrics should consider an instance's difficulty.

\section{Acknowledgments}

We would like to thank all reviewers for their thoughtful comments and encouraging feedback, and Matthew Peters for providing KnowBERT predictions on TACRED. We would also like to thank Elif Kara, Ulli Strohriegel and Tatjana Zeen for the annotation of the dataset. This work has been supported by the German Federal Ministry of Education and Research as part of the projects DEEPLEE (01IW17001) and BBDC2 (01IS18025E), and by the German Federal Ministry for Economic Affairs and Energy as part of the project PLASS (01MD19003E).

\section{References}

Christoph Alt, Marc Hübner, and Leonhard Hennig. 2019. Improving relation extraction by pre-trained language representations. In Proceedings of the 2019 Conference on Automated Knowledge Base Construction, Amherst, Massachusetts.

Livio Baldini Soares, Nicholas FitzGerald, Jeffrey Ling, and Tom Kwiatkowski. 2019. Matching the blanks: Distributional similarity for relation learning. In Proceedings of the 57th Annual Meeting of the Association for Computational Linguistics, pages 2895-2905, Florence, Italy. Association for Computational Linguistics.

Jeremy Barnes, Lilja Øvrelid, and Erik Velldal. 2019. Sentiment analysis is not solved! assessing and probing sentiment classification. In Proceedings of the 2019 ACL Workshop BlackboxNLP: Analyzing and Interpreting Neural Networks for NLP, pages 12-23, Florence, Italy. Association for Computational Linguistics.

Danqi Chen, Jason Bolton, and Christopher D. Manning. 2016. A thorough examination of the CNN/daily mail reading comprehension task. In Proceedings of the 54th Annual Meeting of the Association for Computational Linguistics (Volume 1: Long Papers), pages 2358-2367, Berlin, Germany. Association for Computational Linguistics.

Alexis Conneau, German Kruszewski, Guillaume Lample, Loïc Barrault, and Marco Baroni. 2018. What you can cram into a single $\$ \&$ !\#* vector: Probing sentence embeddings for linguistic properties. In Proceedings of the 56th Annual Meeting of the Association for Computational Linguistics (Volume 1: Long Papers), pages 2126-2136, Melbourne, Australia. Association for Computational Linguistics.

Jacob Devlin, Ming-Wei Chang, Kenton Lee, and Kristina Toutanova. 2019. BERT: Pre-training of 
deep bidirectional transformers for language understanding. In Proceedings of the 2019 Conference of the North American Chapter of the Association for Computational Linguistics: Human Language Technologies, Volume 1 (Long and Short Papers), pages 4171-4186, Minneapolis, Minnesota. Association for Computational Linguistics.

David Harbecke, Robert Schwarzenberg, and Christoph Alt. 2018. Learning explanations from language data. In Proceedings of the 2018 EMNLP Workshop BlackboxNLP: Analyzing and Interpreting Neural Networks for NLP, pages 316-318, Brussels, Belgium. Association for Computational Linguistics.

Iris Hendrickx, Su Nam Kim, Zornitsa Kozareva, Preslav Nakov, Diarmuid Ó Séaghdha, Sebastian Padó, Marco Pennacchiotti, Lorenza Romano, and Stan Szpakowicz. 2010. SemEval-2010 task 8: Multi-way classification of semantic relations between pairs of nominals. In Proceedings of the 5th International Workshop on Semantic Evaluation, pages 33-38, Uppsala, Sweden. Association for Computational Linguistics.

Heng Ji and Ralph Grishman. 2011. Knowledge base population: Successful approaches and challenges. In Proceedings of the 49th Annual Meeting of the Association for Computational Linguistics: Human Language Technologies - Volume 1, HLT'11, pages 1148-1158, Stroudsburg, PA, USA. Association for Computational Linguistics.

Robin Jia and Percy Liang. 2017. Adversarial examples for evaluating reading comprehension systems. In Proceedings of the 2017 Conference on Empirical Methods in Natural Language Processing, pages 2021-2031, Copenhagen, Denmark. Association for Computational Linguistics.

Mandar Joshi, Danqi Chen, Yinhan Liu, Daniel S. Weld, Luke S. Zettlemoyer, and Omer Levy. 2019. Spanbert: Improving pre-training by representing and predicting spans. ArXiv, abs/1907.10529.

Najoung Kim, Roma Patel, Adam Poliak, Patrick Xia, Alex Wang, Tom McCoy, Ian Tenney, Alexis Ross, Tal Linzen, Benjamin Van Durme, Samuel R. Bowman, and Ellie Pavlick. 2019. Probing what different NLP tasks teach machines about function word comprehension. In Proceedings of the Eighth Joint Conference on Lexical and Computational Semantics (*SEM 2019), pages 235-249, Minneapolis, Minnesota. Association for Computational Linguistics.

Tom McCoy, Ellie Pavlick, and Tal Linzen. 2019. Right for the wrong reasons: Diagnosing syntactic heuristics in natural language inference. In Proceedings of the 57th Annual Meeting of the Association for Computational Linguistics, pages 3428-3448, Florence, Italy. Association for Computational Linguistics.
Thien Huu Nguyen and Ralph Grishman. 2015. Relation extraction: Perspective from convolutional neural networks. In Proceedings of the 1st Workshop on Vector Space Modeling for Natural Language Processing, pages 39-48, Denver, Colorado. Association for Computational Linguistics.

Timothy Niven and Hung-Yu Kao. 2019. Probing neural network comprehension of natural language arguments. In Proceedings of the 57th Annual Meeting of the Association for Computational Linguistics, pages 4658-4664, Florence, Italy. Association for Computational Linguistics.

Jeffrey Pennington, Richard Socher, and Christopher Manning. 2014. Glove: Global vectors for word representation. In Proceedings of the 2014 Conference on Empirical Methods in Natural Language Processing (EMNLP), pages 1532-1543, Doha, Qatar. Association for Computational Linguistics.

Matthew E. Peters, Mark Neumann, Robert Logan, Roy Schwartz, Vidur Joshi, Sameer Singh, and Noah A. Smith. 2019. Knowledge enhanced contextual word representations. In Proceedings of the 2019 Conference on Empirical Methods in Natural Language Processing and the 9th International Joint Conference on Natural Language Processing (EMNLP. IJCNLP), pages 43-54, Hong Kong, China. Association for Computational Linguistics.

Alec Radford, Karthik Narasimhan, Tim Salimans, and Ilya Sutskever. 2018. Improving language understanding by generative pre-training. available as a preprint.

Marco Tulio Ribeiro, Sameer Singh, and Carlos Guestrin. 2018. Semantically equivalent adversarial rules for debugging NLP models. In Proceedings of the 56th Annual Meeting of the Association for Computational Linguistics (Volume 1: Long Papers), pages 856-865, Melbourne, Australia. Association for Computational Linguistics.

Sebastian Riedel, Limin Yao, and Andrew McCallum. 2010. Modeling Relations and Their Mentions without Labeled Text. In Proceedings of the European Conference on Machine Learning and Knowledge Discovery in Databases (ECML PKDD '10).

Peng Shi and Jimmy Lin. 2019. Simple bert models for relation extraction and semantic role labeling. ArXiv, abs/1904.05255.

Tongshuang Wu, Marco Tulio Ribeiro, Jeffrey Heer, and Daniel Weld. 2019. Errudite: Scalable, reproducible, and testable error analysis. In Proceedings of the 57th Annual Meeting of the Association for Computational Linguistics, pages 747-763, Florence, Italy. Association for Computational Linguistics.

Kun Xu, Siva Reddy, Yansong Feng, Songfang Huang, and Dongyan Zhao. 2016. Question answering on freebase via relation extraction and textual evidence. 
In Proceedings of the 54th Annual Meeting of the Association for Computational Linguistics (Volume 1: Long Papers), pages 2326-2336. Association for Computational Linguistics.

Daojian Zeng, Kang Liu, Siwei Lai, Guangyou Zhou, and Jun Zhao. 2014. Relation classification via convolutional deep neural network. In Proceedings of COLING 2014, the 25th International Conference on Computational Linguistics: Technical Papers, pages 2335-2344, Dublin, Ireland. Dublin City University and Association for Computational Linguistics.

Yuhao Zhang, Peng Qi, and Christopher D. Manning. 2018. Graph convolution over pruned dependency trees improves relation extraction. In Proceedings of the 2018 Conference on Empirical Methods in Natural Language Processing, pages 2205-2215, Brussels, Belgium. Association for Computational Linguistics.

Yuhao Zhang, Victor Zhong, Danqi Chen, Gabor Angeli, and Christopher D. Manning. 2017. Positionaware attention and supervised data improve slot filling. In Proceedings of the 2017 Conference on Empirical Methods in Natural Language Processing, pages 35-45, Copenhagen, Denmark. Association for Computational Linguistics.

Zhengyan Zhang, Xu Han, Zhiyuan Liu, Xin Jiang, Maosong Sun, and Qun Liu. 2019. ERNIE: Enhanced language representation with informative entities. In Proceedings of the 57th Annual Meeting of the Association for Computational Linguistics, pages 1441-1451, Florence, Italy. Association for Computational Linguistics.

Luisa M. Zintgraf, Taco S. Cohen, Tameem Adel, and Max Welling. 2017. Visualizing deep neural network decisions: Prediction difference analysis. International Conference on Learning Representations.

\section{A Appendix}

\section{A.1 Hyperparameters}

CNN For training we use the hyperparameters of Zhang et al. (2017). We employ Adagrad as an optimizer, with an initial learning rate of 0.1 and run training for 50 epochs. Starting from the 15th epoch, we gradually decrease the learning rate by a factor of 0.9. For the CNN we use 500 filters of sizes $[2,3,4,5]$ and apply $l_{2}$ regularization with a coefficient of $10^{-3}$ to all filter weights. We use tanh as activation and apply dropout on the encoder output with a probability of 0.5 . We use the same hyperparameters for variants with ELMo. For variants with BERT, we use an initial learning rate of 0.01 and decrease the learning rate by a factor of 0.9 every time the validation F1 score is plateauing. Also we use 200 filters of sizes [2, 3, 4, 5].

LSTM/Bi-LSTM For training we use the hyperparameters of Zhang et al. (2017). We employ Adagrad with an initial learning rate of 0.01 , train for 30 epochs and gradually decrease the learning rate by a factor of 0.9 , starting from the 15 th epoch. We use word dropout of 0.04 and recurrent dropout of 0.5. The BiLSTM consists of two layers of hidden dimension 500 for each direction. For training with ELMo and BERT we decrease the learning rate by a factor of 0.9 every time the validation F1 score is plateauing.

GCN We reuse the hyperparameters of Zhang et al. (2018). We employ $S G D$ as optimizer with an initial learning rate of 0.3 , which is reduced by a factor of 0.9 every time the validation F1 score plateaus. We use dropout of 0.5 between all but the last GCN layer, word dropout of 0.04, and embedding and encoder dropout of 0.5. Similar to the authors we use path-centric pruning with $\mathrm{K}=1$. We use two 200-dimensional GCN layers and similar two 200-dimensional feedforward layers with ReLU activation.

Self-Attention After hyperparameter tuning we found 8 layers of multi-headed self-attention to perform best. Each layer uses 8 attention heads with attention dropout of 0.1 , keys and values are projected to 256 dimensions before computing the similarity and aggregated in a feedforward layer with 512 dimensions. For training we use Adam optimizer with an initial learning rate of $10^{-4}$, which is reduced by a factor of 0.9 every time the validation F1 score plateaus. In addition we use word dropout of 0.04, embedding dropout of 0.5 , and encoder dropout of 0.5 .

\section{A.2 Relation Extraction Performance}

Table 6 show the relation extraction performances for the models on TACRED and our revised version. Models with 'w/synt/sem' use named entity and part-of-speech embeddings in addition to the input word embeddings. 


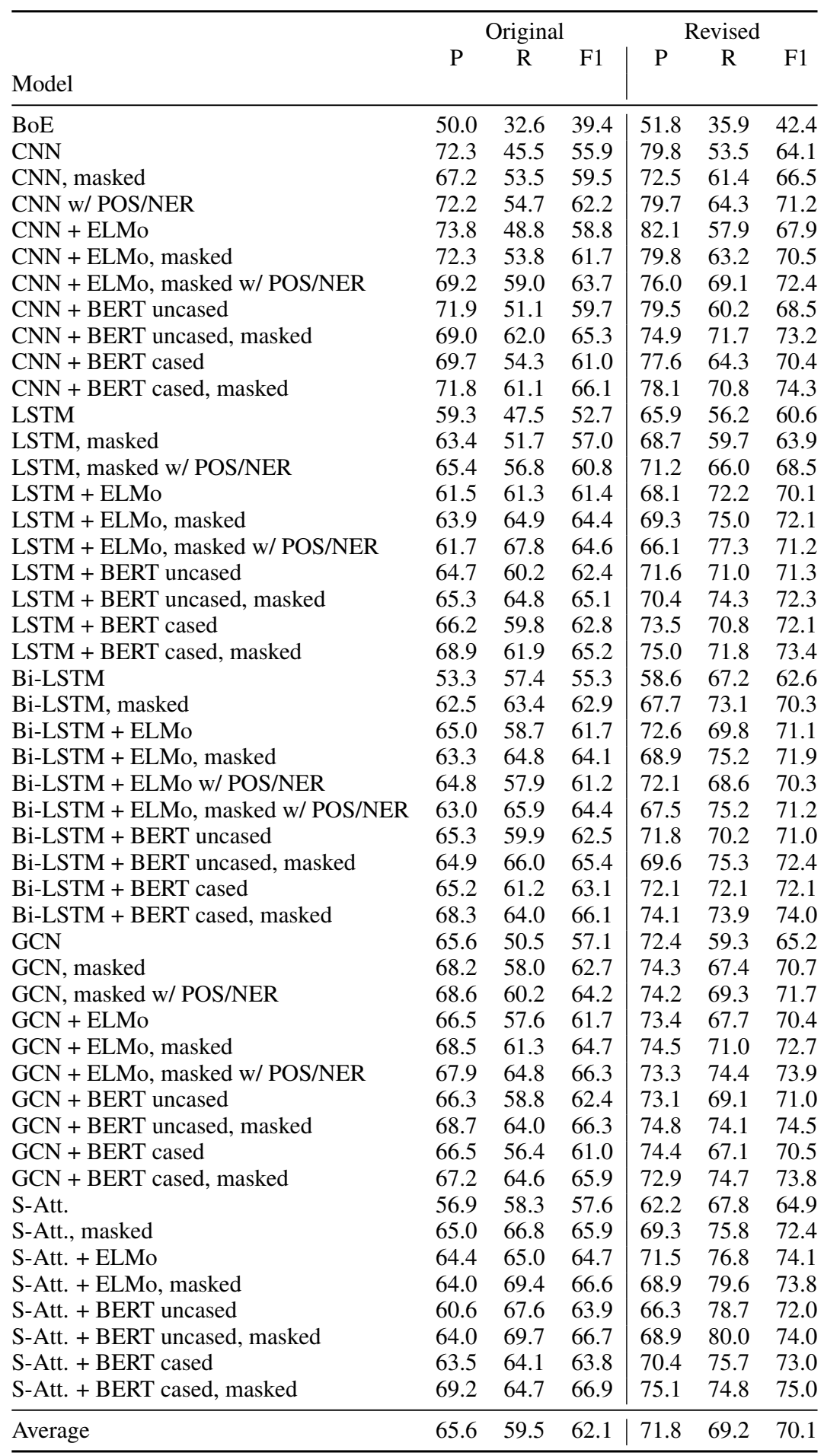

Table 6: Test set performance on TACRED and the revised version for all 49 models we used to select the most challenging instances. We use the same entity masking strategy as Zhang et al. (2017), replacing each entity in the original sentence with a special $<\mathrm{NER}>-\{$ SUBJ, OBJ $\}$ token where $<\mathrm{NER}>$ is the corresponding NER tag. For models w/ POS/NER we concatenate part-of-speech and named entity tag embeddings to each input token embedding. 